\title{
THE CATENARIAN PROPERTY OF POWER SERIES RINGS OVER A PRÜFER DOMAIN
}

\author{
J. T. ARNOLD
}

\begin{abstract}
Let $D$ be a Prüfer domain that has the SFT-property. It is shown that the power series ring $D[[x]]$ is catenarian. If $n>1$ and $\operatorname{dim} D>1$ then the ring $D\left[\left[x_{1}, \ldots, x_{n}\right]\right]$ is not catenarian.
\end{abstract}

1. Introduction. Recall that a ring $R$ is a catenary ring if for each saturated chain $P_{0}<P_{1}<\cdots<P_{n}$ of prime ideals of $R$ we have $\operatorname{rank}\left(P_{n} / P_{0}\right)=n$. In particular, an integral domain $D$ is catenarian if, for every prime ideal $P$ of $D$, the length of every saturated chain of prime ideals between (0) and $P$ equals rank $P$. Based on the work of Ratliff in [9] and Seydi in [10], Lequain has shown in [7] that if $D$ is a Noetherian integral domain then the domains $D[x], D\left[x_{1}, \ldots, x_{n}\right], D[[x]]$, and $D\left[\left[x_{1}, \ldots, x_{n}\right]\right]$ are simultaneously catenarian.

In [4] and [5] it is shown that if $D$ is a finite-dimensional Bezout domain then $D[x]$ is catenarian. More recently, De Souza Doering and Lequain have shown in [8] that if $D$ is a finite dimensional Prüfer domain then $D[x]$ is catenarian. Finally, Bouvier and Fontana have shown in [6] that if $D$ is a locally finite-dimensional Prüfer domain then $D\left[x_{1}, \ldots, x_{n}\right]$ is catenarian for each $n \geq 1$. In view of the above referenced results for Noetherian integral domains, it is natural to inquire about the catenarian properties of $D[[x]]$ and $D\left[\left[x_{1}, \ldots, x_{n}\right]\right]$ when $D$ is a Prüfer domain.

In [1] the author has defined an ideal $A$ of a ring $R$ to be of strong finite type (or an SFT-ideal) provided there is a finitely generated ideal $B \subset A$ and a positive integer $k$ such that $a^{k} \in B$ for each $a \in A$. The ring $R$ satisfies the SFT-property provided each ideal of $R$ is an SFT-ideal. It is shown in [1] (cf. the proof of [1, Theorem 1]) that if a ring $R$ does not have the SFT-property then there exists an infinite chain of prime ideals in $R[[X]]$. We must, therefore, restrict our attention to Prüfer domains with the SFT-property. Such domains have already been studied in [2 and 3].

2. The catenarian property in $D[[x]]$. Throughout this section, $D$ denotes a Prüfer domain with the SFT-property and $K$ denotes the quotient field of $D$. We let $X_{n}=\left\{x_{1}, \ldots, x_{n}\right\}$ and we write $D\left[\left[X_{n}\right]\right]$ to denote $D\left[\left[x_{1}, \ldots, x_{n}\right]\right]$.

LEMMA 1. Let $Q_{1}$ and $Q_{2}$ be prime ideals of $D\left[\left[X_{n}\right]\right]$ such that $Q_{2}<Q_{1}$, $\operatorname{rank}\left(Q_{1} / Q_{2}\right)=1$, and $Q_{i} \cap D=P_{i}, i=1,2$. Then either $P_{1}=P_{2}$ or $\operatorname{rank}\left(P_{1} / P_{2}\right)$ $=1$ and $Q_{1} \subset P_{1}+\left(X_{n}\right)$.

ProOF. Suppose that $P_{2}<P_{1}$, let $p \in P_{1} \backslash P_{1}^{2}$ (cf. [2, Lemma 2.7]), and let $W$ be a valuation overring of $D\left[\left[X_{n}\right]\right]$ with prime ideals $N_{2}<N_{1}$ such that $N_{i} \cap D\left[\left[X_{n}\right]\right]=$ $Q_{i}, i=1,2, N_{1}$ is maximal in $W$, and $N_{1}=\operatorname{rad}(p W)$. Since $V=W \cap K$ is a

Received by the editors October 31, 1984.

1980 Mathematics Subject Classification. Primary 13C15, 13F25; Secondary 13F05.

Key words and phrases. Catenary ring, power series ring, Prüfer domain, prime ideal, rank. 
valuation overring of $D$ centered on $P_{1}$ we must have $V=D_{P_{1}}$. Assume there exists a prime ideal $P$ of $D$ such that $P_{2}<P<P_{1}$. Then $P V \subset \bigcap_{k=1}^{\infty} p^{k} V$ so $P W \subset \bigcap_{k=1}^{\infty} p^{k} W$. If we set $N=\bigcap_{k=1}^{\infty} p^{k} W$ then $N$ is a prime ideal of $W$ and, since $P \subset N \cap D<P_{1}$, it follows that $Q_{2}<N \cap D\left[\left[X_{n}\right]\right]<Q_{1}$. But this contradicts our assumption that $\operatorname{rank}\left(Q_{1} / Q_{2}\right)=1$, so we conclude that $\operatorname{rank}\left(P_{1} / P_{2}\right)=1$.

Let $S$ denote the set of invertible ideals $A$ of $D$ such that $P_{1}<A$. As in $[3$, p. 901], we see that $S$ is a multiplicatively closed set of invertible ideals and $D_{S}=\{\xi \in K \mid \xi A \subset D$ for some $A \in S\}$ is a flat overring of $D$ with prime ideals $\left\{P D_{S} \mid P\right.$ is a prime ideal of $D$ and $\left.P \ngtr P_{1}\right\}$. In particular, $P_{1} D_{S}$ is a maximal ideal of $D_{S}$ and, since $P_{1} D_{S} \cap D=P_{1}$ and $P_{1} D_{S} \subset D$, we have $P_{1}=P_{1} D_{S}$. Following the proof of $\left[\mathbf{3}\right.$, Theorem 3.6] we see that $W \supset D_{s}\left[\left[X_{n}\right]\right]$. Set $P_{1}=N_{1} \cap D_{S}\left[\left[X_{n}\right]\right]$. Then $P_{1} \cap D_{S}=P_{1} D_{S}$, so $P_{1} \subset\left[P_{1}+\left(X_{n}\right)\right] D_{S}\left[\left[X_{n}\right]\right]$. Therefore, $Q_{1}=\mathcal{P}_{1} \cap D\left[\left[X_{n}\right]\right] \subset P_{1}+\left(X_{n}\right)$.

For a prime ideal $Q$ of $D[[x]]$, let $A(Q)$ denote the set of prime ideals $Q^{\prime}$ of $D[[x]]$ such that $Q<Q^{\prime}$ and $\operatorname{rank}\left(Q^{\prime} / Q\right)=1$. Similarly, $B(Q)$ denotes the set of prime ideals $Q^{\prime}$ of $D[[x]]$ such that $Q^{\prime}<Q$ and $\operatorname{rank}\left(Q / Q^{\prime}\right)=1$. Finally, $I(Q)$ denotes the ideal of $D$ consisting of the constant terms of elements of $Q$.

LEMMA 2. Let $Q$ be a prime ideal of $D[[x]]$ such that $Q \cap D=P, P[[x]]<Q$, and $Q \neq P+(x)$. Then

(1) $A(Q)$ is a finite set and $A(Q)=\left\{P^{\prime}+(x) \mid P^{\prime}\right.$ is minimal over $\left.I(Q)\right\} \subset$ $\left\{P^{\prime}+(x) \mid \operatorname{rank}\left(P^{\prime} / P\right)=1\right\}$ and

(2) $P[[x]]$ is the unique element in $B(Q)$.

Proof. Let $Q^{\prime}$ be a prime ideal of $D[[x]]$ and set $P^{\prime}=Q^{\prime} \cap D$. Then $P^{\prime}[[x]] \subset Q^{\prime}$ by $\left[\mathbf{1}\right.$, Theorem 1] and, by applying Lemma 3.5 of $[\mathbf{3}]$ to $\left(D / P^{\prime}\right)[[x]]$, we see that either $Q^{\prime}=P^{\prime}[[x]]$ or $\operatorname{rank}\left(Q^{\prime} / P^{\prime}[[x]]\right)=1$. If $Q^{\prime} \in \mathcal{A}(Q)$ it follows that $P<P^{\prime}$, so, by Lemma $1, \operatorname{rank}\left(P^{\prime} / P\right)=1$ and $Q^{\prime} \subset P^{\prime}+(x)$. But $Q^{\prime} \neq P^{\prime}[[x]]$ by $[2$, Corollary 3.6] so $Q^{\prime}=P^{\prime}+(x)$. Since $Q<Q^{\prime}$ it is necessary that $I(Q) \subset P^{\prime}$. But $I(Q) \not \subset P$ (otherwise we would have $Q \subset P+(x)$ ), so $P^{\prime}$ is minimal over $I(Q)$.

Conversely, let $P^{\prime}$ be a prime ideal of $D$ such that $P^{\prime}$ is minimal over $I(Q)$. Then $Q<P^{\prime}+(x)$, so there exists a prime ideal $Q^{\prime \prime}$ of $D[[x]]$ such that $Q<Q^{\prime \prime} \subset P^{\prime}+(x)$ and $Q^{\prime \prime} \in A(Q)$. As we have just shown, $Q^{\prime \prime}=P^{\prime \prime}+(x)$ for some prime ideal $P^{\prime \prime}$ of $D$ such that $\operatorname{rank}\left(P^{\prime \prime} / P\right)=1$ and $P^{\prime \prime}$ is minimal over $I(Q)$. It follows that $P^{\prime}=P^{\prime \prime}$ and, hence, that $P^{\prime}+(x) \in A(Q)$. The finiteness of $A(Q)$ follows from Corollary 2.6 of $[\mathbf{2}]$.

Now suppose that $Q^{\prime} \in B(Q)$ and $P^{\prime}=Q^{\prime} \cap D$. Since $Q \not \subset P+(x)$, it follows from Lemma 1 that $P=P^{\prime}$. Consequently, $Q^{\prime}=P[[x]]$.

LEMMA 3. Let $P$ be a nonzero prime ideal of $D$ and let $(0)<Q_{1}<\cdots<Q_{s}<$ $P+(x)$ be a saturated chain of prime ideals in $D[[x]]$. Then $s=\operatorname{rank} P$.

ProOF. Suppose that $s>1$ and let $P_{1}$ be the unique rank one prime ideal of $D$ that is contained in $P$. Either $Q_{1}=P_{1}[[x]]$ or $Q_{1} \cap D=(0)$. Since $Q_{2} \cap D \neq(0)$ by [3, Lemma 3.5], $Q_{1} \cap D=(0)$ implies, by Lemma 1 , that $Q_{2} \cap D=P_{1}$. Thus, since $\operatorname{rank} P_{1}[[x]]=1$, in either case we have that $Q_{2}>P_{1}[[x]]$. Since $P+(x)>Q_{2}$ by assumption, it follows that $P \neq P_{1}$; that is, $\operatorname{rank} P \geq 2$. Thus, if $\operatorname{rank} P=1$, then $s=1$. On the other hand, if $\operatorname{rank} P>1$ we may pass to $D / P_{1}$ and argue, by induction, that $s=\operatorname{rank} P$. 
THEOREM 4. If $D$ is a Prüfer domain with the SFT-property, then $D[[x]]$ is catenarian.

ProOF. Let $Q$ be a nonzero prime ideal of $D[[x]]$ with $P=Q \cap D$. If $Q=P[[x]]$ then, by [2, Corollary 3.6], there is a unique chain of prime ideals between (0) and $Q$. Likewise, if $Q>P[[x]]$ but $Q \neq P+(x)$, then, by Lemma 2(2) and [2, Corollary 3.6], there is a unique chain of prime ideals between $(0)$ and $Q$. The remaining case, $Q=P+(x)$, has already been handled in Lemma 3 .

If $\operatorname{dim} D=1$ then $D$ is a Dedekind domain (cf. [2, p. 4]) and hence, by $[\mathbf{7}$, Theorem 2.6], $D\left[\left[X_{n}\right]\right]$ is catenarian for each $n$. If $\operatorname{dim} D=m$ then, by $[3$, Theorem 3.6], $\operatorname{dim} D\left[\left[X_{n}\right]\right]=m n+1$. Indeed, if $M$ is a maximal ideal of $D$ and $\operatorname{rank} M=m$, then $\operatorname{rank}\left(M+\left(X_{n}\right)\right)=m n+1$. But $\operatorname{rank}\left(x_{n}\right)=1$ and

$$
\operatorname{rank}\left[\left(M+\left(X_{n}\right)\right) /\left(x_{n}\right)\right]=\operatorname{rank}\left(M+\left(X_{n-1}\right)\right)=m(n-1)+1,
$$

so if $m>1$ then each saturated chain of the form $(0)<\left(x_{n}\right)<\cdots<M+\left(X_{n}\right)$ has length at most $m(n-1)+2<m n+1$. Thus, $D\left[\left[X_{n}\right]\right]$ is not catenarian.

3. The valuation ring case. A valuation ring $V$ has the SFT-property if and only if it is a discrete valuation ring. (A valuation ring $V$ is discrete provided $P \neq P^{2}$ for each prime ideal $P$ of $V$. Cf. Lemma 2.7 and Proposition 3.1 of $[2]$.) The results of $\S 2$ allow us to give a fairly complete description of the prime spectrum of $V[[x]]$ when $V$ is a discrete valuation ring. First, we restate and strengthen Lemma 2 as follows.

PROPOSITION 5. Let $V$ be a discrete valuation ring and let $Q$ be a prime ideal of $V[[x]]$. If $Q \cap V=P, P[[x]]<Q$, and $Q \neq P+(x)$, then

(1) $\mathcal{A}(Q)=\left\{P^{\prime}+(x)\right\}$ where $P^{\prime}$ is the unique prime ideal of $V$ such that $P<P^{\prime}$ and $\operatorname{rank}\left(P^{\prime} / P\right)=1$,

(2) $B(Q)=\{P[[x]]\}$,

(3) $Q$ is principal.

ProOF. (1) and (2) are immediate from Lemma 2. We first prove (3) for the case $P=(0)$. In this case $\operatorname{rank} P^{\prime}=1$, and if we set $W=V_{P^{\prime}}$ then $W[[x]]_{W \backslash(0)}=$ $V[[x]]_{V \backslash(0)}$. It follows that $Q^{\prime}=Q V[[x]]_{V \backslash(0)} \cap W[[x]]$ is a prime ideal of $W[[x]]$ such that $Q^{\prime} \cap W=(0)$ and $Q^{\prime} \cap V[[x]]=Q$. But $W[[x]]$ is a unique factorization domain so $Q^{\prime}$ is principal.

Suppose that $Q^{\prime}=f^{\prime} W[[x]]$, where $f^{\prime}=\sum_{i=0}^{\infty} w_{i} x^{i}$. Since $Q^{\prime} \not \subset\left(P^{\prime} W\right)[[x]]$, some $w_{i}, i \geq 1$, is a unit in $W$. Thus, we may write $f^{\prime}=f_{1}^{\prime}+x^{k} u$ where $k \geq 1$, $f_{1}^{\prime} \in\left(P^{\prime} W\right)[[x]]$, and $u$ is a unit in $W[[x]]$. If $f=f^{\prime} u^{-1}=f_{1}^{\prime} u^{-1}+x^{k}=f_{1}+x^{k}$ then $f_{1}=f_{1}^{\prime} u^{-1}$ is in $\left(P^{\prime} W\right)[[x]]=P^{\prime}[[x]] \subset V[[x]]$. Therefore, $f \in V[[x]]$ and $Q^{\prime}=f W[[x]]$. Let $q \in Q$ and write $q=f h$ where $h \in W[[x]]$. Then $q=f_{1} h+x^{k} h$ and $f_{1} h \in P^{\prime}[[x]] \subset V[[x]]$ so $x^{k} h=q-f_{1} h \in V[[x]]$. It follows that $h \in V[[x]]$ and $Q=f V[[x]]$.

In the general case we may now conclude that $Q=P[[x]]+f V[[x]]$ where, if $f=\sum_{i=0}^{\infty} a_{i} x^{i}$, then $a_{0} \notin P$. Thus, if $p \in P$ then $p / a_{0}^{n} \in V$ for each positive integer $n$. It follows that $P f^{-1} \subset V[[x]]$ and, hence, that $P[[x]] \subset f V[[x]]$. Therefore, $Q=f V[[x]]$.

QUESTION. What is a characterization of the prime elements of $V[[x]]$ ? 
We conclude with a diagram which illustrates the prime spectrum of a threedimensional discrete valuation ring $V$. Let $(0)=P_{0}<P_{1}<P_{2}<P_{3}$ be the prime ideals of $V$ and let $f_{i}, 0 \leq i \leq 2$, denote arbitrary prime elements of $V[[x]]$ such that $f_{i} V[[x]] \cap V=P_{i}$. We use $A \rightarrow B$ to mean $A>B$. We thus have the following diagram of prime ideals in $V[[x]]$.

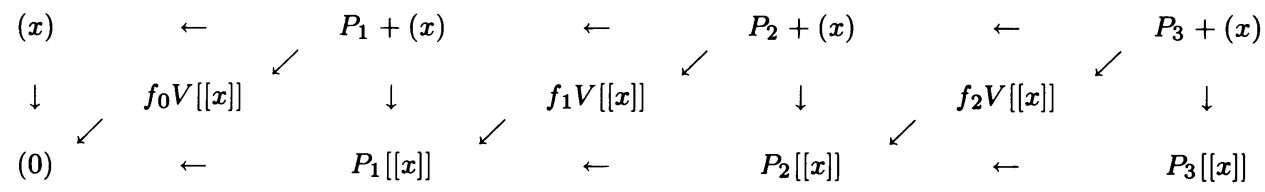

\section{REFERENCES}

1. J. Arnold, Krull dimension in power series rings, Trans. Amer. Math. Soc. 177 (1973), 299-304.

2. __ Power series rings over Prüfer domains, Pacific J. Math. 44 (1973), 1-11.

3. __ Power series rings with finite Krull dimension, Indiana Univ. Math. J. 31 (1982), 897-911.

4. A. Bouvier, F. Burq and G. Germain, Un exemple d'anneau caténaire, Publ. Dép. Math. (Lyon) 17 (1980), 97-101.

5. A. Bouvier, M. Contessa and P. Ribenboim, On chains of prime ideals in polynomial rings, C. R. Math. Rep. Acad. Sci. Canada 3 (1981), 87-92.

6. A. Bouvier and M. Fontana, The catenarian property of the polynomial rings over a Prüfer domain, Preprint.

7. Y. Lequain, Catenarian property in a domain of formal power series, J. Algebra 65 (1980), 110-117.

8. A. M. De Souza Doering and Y. Lequain, Chains of prime ideals in polynomial rings, J. Algebra 78 (1982), 163-180.

9. L. Ratliff, On quasi-unmixed local domains, the altitude formula, and the chain condition for prime ideals. I, Amer. J. Math. 93 (1971), 1070-1108.

10. H. Seydi, Anneaux henséliens et conditions de chaines, Bull. Soc. Math. France 98 (1970), 9-31.

Department of Mathematics, Virginia Polytechnic institute and State UNIVERSITY, BLACKSBURG, VIRGINIA 24061 